\title{
TRANSITIVE FACTORIZATIONS IN THE HYPEROCTAHEDRAL GROUP
}

\author{
GILBERTO BINI* , IAN P. GOULDEN ${ }^{\dagger}$, AND DAVID M. JACKSON ${ }^{\ddagger}$
}

\begin{abstract}
The classical Hurwitz Enumeration Problem has a presentation in terms of transitive factorisations in the symmetric group. This presentation suggests a generalization from type $A$ to other Enite reaection groups and, in particular, to type $B$. We study this generalization both from a combinatorial and a geometric point of view, with the prospect of providing a means of understanding more of the structure of the moduli spaces of maps with an $\mathfrak{S}_{2}$-symmetry. The type $A$ case has been well studied and connects Hurwitz numbers to the moduli space of curves. We conjecture an analogous setting for the type $B$ case that is studied here.
\end{abstract}

\section{INTRODUCTION}

Transitive factorizations of permutations into transpositions occur in Hurwitz's approach to determining the Hurwitz number $h_{g}(\theta)$, the number of genus $g$ ramifed covers of the sphere with elementary branching at a prescribed number of points and arbitrary rami£cation over in£nity speci£ed by the partition $\theta$. This problem, which is called Hurwitz's Enumeration Problem, and its generalizations, have attracted considerable attention in recent years and have been shown to have deep connections through geometry to the moduli space of maps. The presentation of Hurwitz's Problem in terms of factorizations of permutations makes it susceptible to approaches from algebraic combinatorics, and these approaches have assisted our understanding of the Problem. The type $A$ setting of the Problem strongly suggests another direction of generalization, namely to other Enite reaection groups. In this paper we study the Hurwitz Problem for type $B$, the hyperoctahedral group, with the purpose of understanding both the combinatorics and the geometry of this generalization. We derive the main result

Date: July 15, 2005.

*Dipartimento di Matematica, Universitádegli Studi di Milano, Milano, Italy; gilberto.bini@mat.unimi.it.

${ }^{\dagger}$ Department of Combinatorics and Optimization, University of Waterloo, Waterloo, Ontario, Canada.;

ipgoulde@math.uwaterloo.ca.

${ }^{\ddagger}$ Department of Combinatorics and Optimization, University of Waterloo, Waterloo, Ontario, Canada.;

dmjackso@math.uwaterloo.ca. 
by combinatorial means and then provide a geometrical explanation of the result so that the connection between the two approaches may be better understood. We surmise that the $\mathfrak{S}_{2}$-action that is present in the type $B$ Hurwitz Problem might yield new moduli spaces of maps with a speci£c $\mathfrak{S}_{2}$-symmetry.

The organization of the paper is as follows (the few undefned terms appearing in this paragraph are defned later). In Section 2, we give the axiomatization of admissible and near-admissible factorizations for the hyperoctahedral Hurwitz numbers in terms of permutation factorizations, and give combinatorial properties of the hyperoctahedral group. In Section 3, we solve the hyperoctahedral analogue of the Hurwitz problem by enumerating an auxiliary set of factorizations called admissible factorizations. This is our main combinatorial result, stated in Theorem 3.4, which expresses the number of such factorizations (denoted by $s_{g, j}(\lambda, \mu)$ where $\lambda, \mu$ are partitions) as an explicit multiple of the number of transitive factorizations (denoted by $c_{g}(\lambda \cup \mu)$ ). By Hurwitz encoding $c_{g}(\lambda \cup \mu)$ is equal to the Hurwitz number $h_{g}(\lambda \cup \mu)$. In Section 4, we consider the geometric interpretation of the hyperoctahedral transitive factorization problem. We begin with the geometric problem equivalent to Hurwitz factorizations that motivated Hurwitz's [H] work. Then we consider an associated geometric problem that is equivalent to admissible factorizations in the hyperoctahedral group, thus translating our main combinatorial result to a geometric result, given as Theorem 4.1. In Section 5, we use geometric means to prove Theorem 4.1, and formulate a conjecture for moduli spaces based on this proof.

\section{PRELiminaries}

We begin by considering factorizations in the symmetric group and then in the hyperoctahedral group.

2.1. The Hurwitz Problem. Let $\mathfrak{S}_{d}$ be the symmetric group acting on the set $\mathcal{N}_{d}=\{1,, \ldots, d\}$. A partition is a weakly ordered list of positive integers $\alpha=\left(\alpha, \ldots, \alpha_{k}\right)$, where $\alpha_{1} \geq \ldots \geq \alpha_{k}$. The integers $\alpha_{1}, \ldots, \alpha_{k}$ are called the parts of the partition $\alpha$, and we denote the number of parts by $l(\alpha)=k$. If $\alpha_{1}+\ldots+\alpha_{k}=d$, then $\alpha$ is a partition of $d$, and we de $£$ ne $|\alpha|$ by $|\alpha|=d$. If $\lambda, \mu$ are partitions, then $\lambda \cup \mu$ denotes the multiset union of $\lambda$ and $\mu$. Let $\mathcal{C}_{\alpha}$ be the conjugacy class of $\mathfrak{S}_{d}$ consisting of all permutations whose disjoint cycle lengths are specifed by the parts of $\alpha$. If $\sigma_{1}, \ldots, \sigma_{r}$ are elements of a group let $\left\langle\sigma_{1}, \ldots, \sigma_{r}\right\rangle$ denote the subgroup of it generated by $\sigma_{1}, \ldots, \sigma_{r}$.

In the (classical) Hurwitz Problem, we consider $k$-tuples $\sigma=\left(\sigma_{1}, \ldots, \sigma_{k}\right)$, subject to the conditions:

HF1. $\sigma_{1}, \ldots, \sigma_{k}$ are transpositions in $\mathfrak{S}_{d}$;

HF2. $\sigma_{1} \cdots \sigma_{k}=\rho$ where $\rho \in \mathcal{C}_{\alpha}$; 
HF3. $\left\langle\sigma_{1}, \ldots, \sigma_{k}\right\rangle$ acts transitively on $\mathcal{N}_{d}$;

HF4. $2 g+d+l(\alpha)-2=k$.

We call a factorization $\sigma$ of $\rho$ satisfying these conditions a Hurwitz factorization. Let

$$
c_{g}(\alpha)
$$

be the number of Hurwitz factorizations of each $\rho \in \mathcal{C}_{\alpha}$. Note that condition HF4 is not a restriction; instead, it de $£$ nes a non-negative integer parameter $g$.

The Hurwitz Problem has been the subject of substantial study in the last decade. Goulden and Jackson [GJ1] have obtained an explicit formula for $c_{g}(\alpha)$ in the case $g=0$, and also in the case $g=1$ (for the latter, see [GJ2], and Vakil [V2]). Ekedahl, Lando, Shapiro and Vainshtein [ELSV] have given an expression for $c_{g}(\alpha)$ as a Hodge integral, for arbitrary $g$.

2.2. The hyperoctahedral group $\mathbb{C H}_{d}$. In this paper, we consider an analogue of the Hurwitz Problem for the hyperoctahedral group. Let $\widehat{\mathfrak{S}}_{d}$ be the symmetric group acting on the set $\widehat{\mathcal{N}}_{d}=\{1, \widehat{1}, \ldots, d, \widehat{d}\}$ and let $\varepsilon=$ $(1 \widehat{1}) \cdots(d \widehat{d})$, in cycle notation. We regard the hyperoctahedral group $\mathfrak{H}_{d}$ as being embedded in $\widehat{\mathfrak{S}}_{d}$ as the centralizer of $\varepsilon$. Now, for $\sigma \in \widehat{\mathfrak{S}}_{d}$, let $\sigma^{\varepsilon}$ denote conjugation of $\sigma$ by $\varepsilon$, so $\sigma^{\varepsilon}=\varepsilon \sigma \varepsilon$. Then for $\pi \in \mathfrak{H}_{d}$, we have $\pi=\pi^{\varepsilon}$. It is useful combinatorially to observe that conjugation of $\pi$ by $\varepsilon$ interchanges the hatted and unhatted symbols of $\widehat{\mathcal{N}}_{d}$. Let $c$ be a cycle in the disjoint cycle representation of $\pi$. Then either $c^{\varepsilon}=c$, in which case we call $c$ an $\varepsilon$-invariant cycle, or there is another cycle of $\pi$ that is equal to $c^{\varepsilon}$, in which case we call $\left(c, c^{\varepsilon}\right)$ an $\varepsilon$-conjugate pair of cycles. If $c$ is an $\varepsilon$-invariant cycle, then $i$ lies on $c$ if and only if $\hat{i}$ lies on $c$, for each $i=1, \ldots, d$. Thus the length of $c$ is even. If $\left(c, c^{\varepsilon}\right)$ is an $\varepsilon$-conjugate pair of cycles then $i$ lies on $c$ if and only if $\hat{i}$ lies on $c^{\varepsilon}$, and $\widehat{i}$ lies on $c$ if and only if $i$ lies on $c^{\varepsilon}$, for each $i=1, \ldots, d$. Thus the lengths of $c$ and $c^{\varepsilon}$ are equal.

Let $\mathcal{A}_{\lambda, \mu}$ be the set of all $\pi \in \mathfrak{H}_{d}$ such that the parts of $2 \lambda$ specify the lengths of the $\varepsilon$-invariant cycles of $\pi$, and the parts of $\mu \cup \mu$ specify the lengths of the cycles in the $\varepsilon$-conjugate pairs of cycles of $\pi$. Clearly, if $\pi \in$ $\mathcal{A}_{\lambda, \mu}$, then $\pi \in \mathcal{C}_{\alpha}$, where $\mathcal{C}_{\alpha}$ is the conjugacy class of $\widehat{\mathfrak{S}}_{d}$ specifed by $\alpha=$ $2 \lambda \cup \mu \cup \mu$. Thus, $l(\lambda)+2 l(\mu)=l(\alpha)$, and $|\lambda|+|\mu|=d$, since $\alpha$ is a partition of $2 d$.

With each $\pi \in \mathfrak{H}_{d}$, we associate a unique $b_{\pi} \in \mathfrak{S}_{d}$, called the source of $\pi$, which is obtained as follows. For each $\varepsilon$-conjugate pair of cycles $\left(c, c^{\varepsilon}\right)$ in $\pi$, if we remove the hats from each occurrence of $\widehat{1}, \ldots, \widehat{d}$, then $c$ and $c^{\varepsilon}$ give the same cycle $c^{\prime}$ of elements in $\mathcal{N}_{d}$. For each $\varepsilon$-invariant cycle $c$, if we remove the hats from each occurrence of $\widehat{1}, \ldots, \widehat{d}$, then $c$ gives a cycle that consists of two concatenated copies of a cycle $c^{\prime}$ of elements in $\mathcal{N}_{d}$. Then 
the disjoint cycles of $b_{\pi}$ consist of all the $c^{\prime}$ obtained from the cycles of $\pi$ as above. For example, if $\pi=(1 \widehat{5} 4)(\widehat{1} 5 \widehat{4})(2 \widehat{3} \widehat{2} 3)$, then $b_{\pi}=(154)(23)$.

There are $2^{d}$ different elements $\pi \in \mathfrak{H}_{d}$ with the same source $\rho=b_{\pi}$, which can be seen as follows. If a cycle in $\rho$ has $m$ elements, then there are 2 choices, $\varepsilon$-invariant cycle or $\varepsilon$-conjugate pair of cycles, for the corresponding cycles in $\pi$. This $£$ xes the location of the smallest element $i$ of the cycle and its mate $\hat{i}$. Then, there are 2 choices for the locations of each of the other $m-1$ elements $j$ and their mates $\hat{j}$. Thus there are $2^{m}$ choices for $\pi$ arising from a cycle of length $m$ in $\rho$, and the product of choices over all the cycles gives the total of $2^{d}$ choices of $\pi$. Moreover, if $\rho \in \mathcal{C}_{\alpha}$, then for each such $\pi$, we have $\pi \in \mathcal{A}_{\lambda, \mu}$, where $\lambda \cup \mu=\alpha$. Of course, it immediately follows that $\left|\mathfrak{H}_{d}\right|=2^{d} d$ !.

2.3. Admissible and near-admissible factorizations. For the hyperoctahedral analogue of the Hurwitz Problem, let $\left.\mathcal{F}_{d}=\{(i j) \widehat{(i j}) \cup(i \widehat{j}) \widehat{(i} j\right) \mid 1 \leq$ $i<j \leq d\}$, and $\mathcal{G}_{d}=\{(j \widehat{j}) \mid 1 \leq j \leq d\}$; these sets, for different reasons, are both analogues in $\mathfrak{H}_{d}$ for transpositions in $\mathfrak{S}_{d}$. Consider $(k+j)$-tuples $a=\left(a_{1}, \ldots, a_{k+j}\right)$, subject to the conditions:

AF1. $a_{1}, \ldots, a_{k+j} \in \mathcal{F}_{d} \cup \mathcal{G}_{d}$, with $k$ of them chosen from $\mathcal{F}_{d}$, and $j$ of them from $\mathcal{G}_{d}$;

AF2. $a_{1} \cdots a_{k+j}=\pi$, where $\pi \in \mathcal{A}_{\lambda, \mu}$, for some $\lambda$, $\mu$ with $|\lambda|+|\mu|=d$;

AF3. $\left\langle a_{1}, \ldots, a_{k+j}\right\rangle$ acts transitively on $\widehat{\mathcal{N}}_{d}$;

AF4. $2 g+2 d+l(\lambda)+2 l(\mu)-2=2 k+j$.

For brevity, we call a factorization $a$ that satis£es these four conditions an admissible factorization of $\pi$, and let

$$
s_{g, j}(\lambda, \mu)
$$

be the number of admissible factorizations of each $\pi \in \mathcal{A}_{\lambda, \mu}$, with $d, j, g$ $£$ xed as above. Again, note that condition AF4 is not a restriction, but deEnes a non-negative integer $g$.

To enumerate admissible factorizations, our strategy is to introduce an associated set of factorizations, called near-admissible factorizations. It is convenient to consider the mapping $R: \mathcal{F}_{d} \cup \mathcal{G}_{d} \rightarrow \mathfrak{S}_{d}$, defned by $R((i j)(\hat{i} \widehat{j}))=$ $R((i \widehat{j})(\widehat{i} j))=(i j)$ for $1 \leq i<j \leq d$, and $R((j \widehat{j}))=\iota$ (the identity in $\left.\mathfrak{S}_{d}\right)$ for $1 \leq j \leq d$. A factorization that satis£es the conditions for admissibility, with condition AF3 replaced by:

NAF3. $\left\langle R\left(a_{1}\right), \ldots, R\left(a_{k+j}\right)\right\rangle$ acts transitively on $\mathcal{N}_{d}$,

is called a near-admissible factorization. Note that condition AF3 implies condition NAF3, but not conversely. For example, $\langle R((1 \widehat{2})(\widehat{1} 2)), R((13)(\widehat{1} \widehat{3}))\rangle$, which is equal to $\langle(12)(13)\rangle$, acts transitively on $\mathcal{N}_{3}$. However, $\langle(1 \widehat{2})(\widehat{1} 2),(13)(\widehat{1} \widehat{3})\rangle$ 
does not act transitively on $\widehat{\mathcal{N}}_{3}$, although it does act transitively on the subsets $\mathcal{R}$ and $\mathcal{R}^{\varepsilon}$ of $\widehat{N}_{3}$ where $\mathcal{R}=\{1, \widehat{2}, 3\}$. Let

$$
t_{g, j}(\lambda, \mu)
$$

be the number of near-admissible factorizations of each $\pi \in \mathcal{A}_{\lambda, \mu}$, with $g, j$ satisfying the four conditions. In particular, we have $2 g+2 d+l(\lambda)+$ $2 l(\mu)-2=2 k+j$. When $j=0$ and $l(\lambda)=0$, it is possible to have $g=-1$. The choice of $g=-1$ should be viewed formally, with $g$ defned by the above equation; the relaxation of condition AF3 to condition NAF3 allows factorizations with fewer factors (smaller choices of $k$ ) to occur, as in the example $(1 \widehat{2})(\widehat{1} 2)(13)(\widehat{1} \widehat{3})$ above.

\section{ENUMERATING FACTORIZATIONS IN THE HYPEROCTAHEDRAL GROUP}

In this section, we enumerate admissible factorizations, by obtaining two relationships between the numbers of admissible, near-admissible and Hurwitz factorizations.

3.1. The Erst relationship. The Erst construction, which follows, is a "doubling" construction applied to Hurwitz factorizations, that creates uniquely the near-admissible factorizations that are not admissible. It includes the explanation of how near-admissible factorizations with $g=-1$ arise. In the proof of this result, some terminology for transitivity is useful. If an element $\theta$ of a subgroup of the symmetric group acting on a set $\mathcal{M}$ maps $i$ to $j$, for $i, j \in \mathcal{M}$ (so $\theta^{-1}$ maps $j$ to $i$ ), and we say (symmetrically) that $i$ and $j$ are in the same component of $\mathcal{M}$. If the subgroup acts transitively on $\mathcal{M}$, then the elements of $\mathcal{M}$ are in a single component of $\mathcal{M}$.

Theorem 3.1. For $g \geq 0$,

$$
t_{2 g-1, j}(\lambda, \mu)-s_{2 g-1, j}(\lambda, \mu)=\left\{\begin{array}{cl}
2^{l(\mu)-1} c_{g}(\mu) & j=0 \text { and } \lambda=\emptyset \\
0 & \text { otherwise. }
\end{array}\right.
$$

Proof: We characterize the near-admissible factorizations that are not admissible. Consider an arbitrary near-admissible factorization. From the form of the factors in $\mathcal{F}_{d}$, condition NAF3 implies that for each $1 \leq i<$ $j \leq d$, either $i, j$ are in the same component and $\widehat{i}, \widehat{j}$ are in the same component, or $i, \widehat{j}$ are in the same component and $\widehat{i}, j$ are in the same component, or both. Thus, for a near-admissible factorization that is not admissible, there must be some set $\mathcal{R}=\left\{r_{1}, \ldots, r_{d}\right\}$, with $r_{1}=1$, and $r_{i}=i$ or $\widehat{i}$ for $i=2, \ldots, d$, such that $\left\{r_{1}, \ldots, r_{d}\right\}$ are all in one component and $\mathcal{R}^{\varepsilon}=\left\{r_{1}, \ldots, r_{d}\right\}^{\varepsilon}$ are all in another component. In particular, this means that $i$ and $\hat{i}$ are in different components for all $i=1, \ldots, d$. But each factor $(i \widehat{i})$ in $\mathcal{G}_{d}$ puts $i$ and $\hat{i}$ in the same component, so this is only possible for 
near-admissible factorizations with $j=0$. Similarly, each $\varepsilon$-invariant cycle contains both $i$ and $\widehat{i}$, for some $i=1, \ldots, d$, so this is only possible for near-admissible factorizations with $\lambda=\emptyset$ (the empty partition).

Now, consider a near-admissible factorization that is not admissible, corresponding to $\mathcal{R}$ as above. Then, this is a factorization $a=\left(a_{1}, \ldots, a_{k}\right)$ of $\pi \in \mathcal{A}_{\emptyset, \mu}$, where $a_{i}=\sigma_{i} \sigma_{i}^{\varepsilon}$, for $i=1, \ldots, k$, and $\sigma_{i}$ is a transposition on $\mathcal{R}$. Moreover, $\pi=\rho \rho^{\varepsilon}$, where $\rho \in \mathcal{C}_{\mu}$ is a permutation on $\mathcal{R}$, since $\pi$ contains only $\varepsilon$-conjugate pairs of cycles $\left(c, c^{\varepsilon}\right)$, where either $c$ or $c^{\varepsilon}$ contains only elements from $\mathcal{R}$, for each such conjugate pair of cycles. Thus each such factorization corresponds to a unique Hurwitz factorization of $\rho \in \mathcal{C}_{\mu}$, in the symmetric group acting on $\mathcal{R}$. But there are $2^{l(\mu)-1}$ choices of $\mathcal{R}$ that are consistent with each such $\pi \in \mathcal{A}_{\emptyset, \mu}$, since, for each $\varepsilon$-conjugate pair of cycles $\left(c, c^{\varepsilon}\right)$ not containing elements $1, \hat{1}$, we have two choices: either the elements of $c$ are placed in $\mathcal{R}$ (and the elements of $c^{\varepsilon}$ are thus in $\mathcal{R}^{\varepsilon}$ ), or the elements of $c^{\varepsilon}$ are placed in $\mathcal{R}$ (and the elements of $c$ are thus in $\mathcal{R}^{\varepsilon}$ ). This gives the factor of $2^{l(\mu)-1}$ on the right hand side of the result.

Now, if the Hurwitz factorization of $\rho$ has genus $g$ and the near-admissible factorization of $\pi$ has genus $g^{\prime}$, then $2 g+d+l(\mu)-2=k$, from condition HF4 for the Hurwitz factorization. But, for the near-admissible factorization, condition AF4 gives $2 g^{\prime}+2 d+2 l(\mu)-2=2 k$, so, by eliminating $k$, we conclude that $g^{\prime}=2 g-1$. The result follows.

Clearly, $g^{\prime}=-1$ when $g=0$, and thus near-admissible factorizations with $g^{\prime}=-1$ arise. Of course, none of these is admissible.

3.2. Some enumerative properties of $\mathbb{C H}_{d}$. We now turn to the second relationship directly relating near-admissible and Hurwitz factorizations. The proof depends on the following results concerning the group algebra $\mathbb{C H}_{d}$ of the hyperoctahedral group. Let $x_{j}=(j \widehat{j})$, for $j=1, \ldots, d$, and

$$
\mathrm{E}_{d}=\sum_{\substack{\alpha \subseteq \mathcal{N}_{d} \\|\alpha| \text { even }}} \prod_{j \in \alpha} x_{j}, \quad \mathrm{O}_{d}=\sum_{\substack{\alpha \subseteq \mathcal{N}_{d} \\|\alpha| \text { odd }}} \prod_{j \in \alpha} x_{j}, \quad \mathrm{~L}_{d}=\sum_{j=1}^{d} x_{j},
$$

where it is noted that $x_{1}, \ldots, x_{d}$ commute.

Proposition 3.2. (1) $\mathrm{E}_{d}, \mathrm{O}_{d}, \mathrm{~L}_{d}$ commute, and

$$
\mathrm{L}_{d} \mathrm{E}_{d}=d \mathrm{O}_{d}, \quad \mathrm{~L}_{d} \mathrm{O}_{d}=d \mathrm{E}_{d} .
$$

(2) Let $\sigma_{i}=\left(u_{i} v_{i}\right)$, for $i=1, \ldots, k$, be transpositions in $\mathfrak{S}_{d}$, where $\left\langle\sigma_{1}, \ldots, \sigma_{k}\right\rangle$ acts transitively on $\mathcal{N}_{d}$. Then

$$
\prod_{i=1}^{k}\left(1+x_{u_{i}} x_{v_{i}}\right)=2^{k-d+1} \mathrm{E}_{d}
$$


(3) If $\rho \in \mathfrak{S}_{d}$, then

$$
\left(\mathrm{E}_{d}+\mathrm{O}_{d}\right) \rho \rho^{\varepsilon}=\sum_{\substack{\pi \in \mathfrak{H}_{d} \\ b_{\pi}=\rho}} \pi .
$$

Proof: (1) Let $\mathbf{x}=\left(x_{1}, \ldots, x_{d}\right), \mathbf{i}=\left(i_{1}, \ldots, i_{d}\right)$, and $\mathbf{x}^{\mathbf{i}}=x_{1}^{i_{1}} \cdots x_{d}^{i_{d}}$. Let 1 denote the (row) vector of $d 1^{\prime}$ 's and let $\mathcal{V}_{d}=\operatorname{ker}(\mathbf{1}) \leq G F(2)^{d}$. Since $x_{1}^{2}=\cdots=x_{d}^{2}=\iota$, then $\mathrm{x}^{\mathrm{i}}$ is a term of $\mathrm{E}_{d}$ if and only if $\mathbf{i} \in \mathcal{V}_{d}$, so

$$
\mathrm{E}_{d}=\sum_{\mathbf{i} \in \mathcal{V}_{d}} \mathbf{x}^{\mathbf{i}}
$$

We Erst prove the result for $k=d-1$. Let $e_{i j}$, for $i \neq j$, be the vector with 1 's in positions $i$ and $j$, and 0 's elsewhere. Then $e_{i j} \in \mathcal{V}_{d}$ for all $i \neq j$ and

$$
\prod_{i=1}^{d-1}\left(1+x_{u_{i}} x_{v_{i}}\right)=\sum \mathbf{x}^{\mathbf{i}},
$$

where the summation is over $\mathbf{i}$ in $\operatorname{span}\left\{e_{u_{1} v_{1}}, \ldots, e_{u_{d-1} v_{d-1}}\right\}$.

We now prove that $\left\{e_{u_{1} v_{1}}, \ldots, e_{u_{d-1} v_{d-1}}\right\}$ is linearly independent. Suppose the contrary. Since the group $\left\langle\sigma_{1}, \ldots, \sigma_{d-1}\right\rangle$ acts transitively on $\mathcal{N}_{d}$, then the graph on vertex-set $\mathcal{N}_{d}$, with edges given by $\left\{u_{1}, v_{1}\right\}, \ldots,\left\{u_{d-1}, v_{d-1}\right\}$ is connected, and is therefore a tree $T$ on $d$ vertices. Then, from the supposition, $T$ has a non-trivial connected subgraph $T^{\prime}$, each of whose vertices has even degree. But $T^{\prime}$ is a tree, and it therefore has at least two vertices of degree one, which is a contradiction. It follows that $\left\{e_{u_{1} v_{1}}, \ldots, e_{u_{d-1} v_{d-1}}\right\}$ is linearly independent. But these are vectors in $\mathcal{V}_{d}$, and $\operatorname{dim} \mathcal{V}_{d}=d-1$. Thus $\operatorname{span}\left\{e_{u_{1} v_{1}}, \ldots, e_{u_{d-1} v_{d-1}}\right\}=\mathcal{V}_{d}$, and the result follows in the case $k=d-1$ from (1) and (2).

To prove the result for all $k$, note that the transitivity of the action of $\left\langle\sigma_{1}, \ldots, \sigma_{k}\right\rangle$ on $\widehat{\mathcal{N}}_{d}$ implies that the graph on vertex-set $\mathcal{N}_{d}$, with edges given by $\left\{u_{1}, v_{1}\right\}, \ldots,\left\{u_{k}, v_{k}\right\}$ is connected, and therefore contains a spanning tree (so $k \geq d-1$ ). Now the terms in the product on the left hand side of the result commute, so the product of the terms corresponding to the $d-1$ edges of any spanning tree is equal to $\mathrm{E}_{d}$, from above. But $x_{j} \mathrm{E}_{d}=\mathrm{O}_{d}$ for $j=1, \ldots, d$, so $x_{i} x_{j} \mathrm{E}_{d}=\mathrm{E}_{d}$ for $1 \leq i, j \leq d$. Thus $\left(1+x_{i} x_{j}\right) \mathrm{E}_{d}=2 \mathrm{E}_{d}$ so each of the remaining $k-(d-1)$ terms contributes an additional factor of 2 . The result follows immediately.

(2) The $x_{j}$ 's commute, and $x_{j}^{2}=\iota$, so $x_{j} \mathrm{E}_{d}=\mathrm{O}_{d}$ and $x_{j} \mathrm{O}_{d}=\mathrm{E}_{d}$, for any $j=1, \ldots, d$, and the result follows.

(3) Consider the cycle $c=\left(c_{1}, \ldots, c_{m}\right)$ in $\rho$ (with the convention that $c_{1}$ is the smallest element on the cycle), and an arbitrary subset $\left\{\alpha_{1}, \ldots, \alpha_{i}\right\}$ of $\{1, \ldots, m\}$, where $1 \leq \alpha_{1}<\cdots<\alpha_{i} \leq m$. Then, if $i$ is even, we obtain

$$
x_{c_{\alpha_{1}}} \cdots x_{c_{\alpha_{i}}} c c^{\varepsilon}=\left(c_{1} \ldots c_{\alpha_{1}-1} \widehat{c}_{\alpha_{1}} \ldots \widehat{c}_{\alpha_{2}-1} c_{\alpha_{2}} \ldots \widehat{c}_{\alpha_{i}-1} c_{\alpha_{i}} \ldots c_{m}\right)
$$




$$
\left(\widehat{c}_{1} \ldots \widehat{c}_{\alpha_{1}-1} c_{\alpha_{1}} \ldots c_{\alpha_{2}-1} \widehat{c}_{\alpha_{2}} \ldots c_{\alpha_{i}-1} \widehat{c}_{\alpha_{i}} \ldots \widehat{c}_{m}\right)
$$

which is an $\varepsilon$-conjugate pair of cycles. However, if $i$ is odd, we obtain

$$
\begin{gathered}
x_{c_{\alpha_{1}}} \ldots x_{c_{\alpha_{i}}} c c^{\varepsilon}=\left(c_{1} \ldots c_{\alpha_{1}-1} \widehat{c}_{\alpha_{1}} \ldots \widehat{c}_{\alpha_{2}-1} c_{\alpha_{2}} \ldots c_{\alpha_{i}-1} \widehat{c}_{\alpha_{i}} \ldots \widehat{c}_{m}\right. \\
\left.\widehat{c}_{1} \ldots \widehat{c}_{\alpha_{1}-1} c_{\alpha_{1}} \ldots c_{\alpha_{2}-1} \widehat{c}_{\alpha_{2}} \ldots \widehat{c}_{\alpha_{i}-1} c_{\alpha_{i}} \ldots c_{m}\right),
\end{gathered}
$$

which is an $\varepsilon$-invariant cycle. Note that these two cases, together, create uniquely all $2^{m}$ choices of cycles in $\pi$ that have $c$ as a source. The result follows, since when each term in $\mathrm{O}_{d}+\mathrm{E}_{d}$ is multiplied by $\rho \rho^{\varepsilon}$, we obtain a product of factors of the type

$$
x_{c_{\alpha_{1}}} \cdots x_{c_{\alpha_{i}}} c c^{\varepsilon}
$$

one for each cycle $c$ of $\rho$.

3.3. The second relationship. The second result, stated below, expresses the number of near-admissible factorizations in the general case directly in terms of the classical Hurwitz numbers.

Theorem 3.3. For $g \geq 0$, with $j \equiv l(\lambda)(\bmod 2)$, then

$$
t_{2 g-1+\frac{j+l(\lambda)}{2}, j}(\lambda, \mu)=\left(\begin{array}{c}
k+j \\
j
\end{array}\right) 2^{k-d+1} d^{j} c_{g}(\lambda \cup \mu)
$$

where

$$
2 g+d+l(\lambda)+l(\mu)-2=k .
$$

Proof: Suppose that $\sigma=\left(\sigma_{1}, \ldots, \sigma_{k}\right)$ is a Hurwitz factorization of $\rho$, where $\sigma_{j}=\left(p_{j} q_{j}\right)$, for $j=1, \ldots, k$. Let $\mathcal{N F}_{j}(\sigma)$ be the set of all near-admissible factorizations $a=\left(a_{1}, \ldots, a_{k+j}\right)$, with $k$ factors from $\mathcal{F}_{d}$, with the restriction that the $k$ transpositions in the list $R\left(a_{1}\right), \ldots, R\left(a_{k+j}\right)$ are, in left to right order, $\sigma_{1}, \ldots, \sigma_{k}$. Let

$$
N F_{j}(\sigma)=\sum_{a \in \mathcal{N F}_{j}(\sigma)} a_{1} \cdots a_{k+j} \in \mathbb{C H}_{d}
$$

In this proof we adopt the convention that $\prod_{j=1}^{k}$ is an iterated non-commutative product, with the terms multiplied from left to right and indexed by $j$ in increasing order. Let the $k$ transpositions from $\mathcal{G}_{d}$ occur in positions $m_{1}, \ldots, m_{k}$, where $1 \leq m_{1}<\cdots<m_{k} \leq k+j$, with the convention that $m_{k+1}=k+j+1$. Then, lifting from the Hurwitz factorizations, we have

$$
\begin{aligned}
N F_{j}(\sigma) & =\sum_{m_{1}, \ldots, m_{k}} \mathrm{~L}_{d}^{m_{1}-1} \prod_{j=1}^{k}\left(\left(p_{j} q_{j}\right)\left(\widehat{p}_{j} \widehat{q}_{j}\right)+\left(p_{j} \widehat{q}_{j}\right)\left(\widehat{p}_{j} q_{j}\right)\right) \mathrm{L}_{d}^{m_{j+1}-m_{j}} \\
& =\mathrm{L}_{d}^{j} \sum_{m_{1}, \ldots, m_{k}} \prod_{j=1}^{k}\left(\left(p_{j} q_{j}\right)\left(\widehat{p}_{j} \widehat{q}_{j}\right)+\left(p_{j} \widehat{q}_{j}\right)\left(\widehat{p}_{j} q_{j}\right)\right) \\
& =\left(\begin{array}{c}
k+j \\
j
\end{array}\right) \mathrm{L}_{d}^{j} \prod_{j=1}^{k}\left(\left(p_{j} q_{j}\right)\left(\widehat{p}_{j} \widehat{q}_{j}\right)+\left(p_{j} \widehat{q}_{j}\right)\left(\widehat{p}_{j} q_{j}\right)\right)
\end{aligned}
$$


since $\mathrm{L}_{d}$ commutes with all elements of the hyperoctahedral group, and there are $\left(\begin{array}{c}k+j \\ j\end{array}\right)$ choices of $m_{1}, \ldots, m_{k}$. But, for $i \neq j$, we have $(i \widehat{j})(\widehat{i} j)=$ $x_{i} x_{j}(i j)(\widehat{i} \widehat{j})$, and $(\widehat{i} \widehat{j})=(i j)^{\varepsilon}$, so $(i j)(\hat{i} \widehat{j})+(i \widehat{j})(\widehat{i} j)=\left(1+x_{i} x_{j}\right)(i j)(i j)^{\varepsilon}$. Applying this equation to rewrite the terms in the above product, we obtain

$$
N F_{j}(\sigma)=\left(\begin{array}{c}
k+j \\
j
\end{array}\right) \mathrm{L}_{d}^{j} \prod_{j=1}^{k}\left(1+x_{p_{j}} x_{q_{j}}\right) \sigma_{j} \sigma_{j}^{\varepsilon} .
$$

To simplify this, note that for $i \neq j$, we have $(i j)(\widehat{i} \widehat{j}) x_{i}=x_{j}(i j)(\widehat{i} \widehat{j})$ and $(i j)(\widehat{i} \hat{j}) x_{m}=x_{m}(i j)(\widehat{i} \widehat{j})$, where $m \neq i, j$. Thus, for $i \neq j$ and all $m$, we have

$\left.(i j)(\widehat{i} \hat{j}) x_{m}=x_{m^{\prime}}(i j) \widehat{(i j}\right)$, where the permutation $(i j)$ on $\mathcal{N}_{d}$ maps $m$ to $m^{\prime}$. Applying this equation to move the factors $1+x_{p_{j}} x_{q_{j}}$ in (3) to the left, we obtain

$$
\begin{aligned}
N F_{j}(\sigma) & =\left(\begin{array}{c}
k+j \\
j
\end{array}\right) \mathrm{L}_{d}^{j}\left(\prod_{j=1}^{k}\left(1+x_{u_{j}} x_{v_{j}}\right)\right) \prod_{j=1}^{k} \sigma_{j} \sigma_{j}^{\varepsilon} \\
& =\left(\begin{array}{c}
k+j \\
j
\end{array}\right) \mathrm{L}_{d}^{j}\left(\prod_{j=1}^{k}\left(1+x_{u_{j}} x_{v_{j}}\right)\right) \rho \rho^{\varepsilon},
\end{aligned}
$$

where $\sigma_{1} \cdots \sigma_{j-1}$ maps $p_{j}$ to $u_{j}$ and maps $q_{j}$ to $v_{j}$, for $j=1, \ldots, k$.

We now prove that $\left\langle\left(u_{1} v_{1}\right), \ldots,\left(u_{k} v_{k}\right)\right\rangle$ acts transitively on $\mathcal{N}_{d}$. For $j=$ $0, \ldots, k$, let $G_{j}$ be the graph on vertex-set $\mathcal{N}_{d}$ with edges $\left\{p_{1}, q_{1}\right\}, \ldots,\left\{p_{j}, q_{j}\right\}$, and $H_{j}$ be the graph on vertex-set $\mathcal{N}_{d}$ with edges $\left\{u_{1}, v_{1}\right\}, \ldots,\left\{u_{j}, v_{j}\right\}$. In this notation, condition HF3 implies that $G_{k}$ is connected, and we must establish that $H_{k}$ is connected. To do so, we prove by induction on $j$, that the (connected) components of $H_{j}$ have the same vertex-sets as the components of $G_{j}$, for each $j=0, \ldots, k$. The result is clearly true for $j=0$, since neither $G_{0}$ nor $H_{0}$ has any edges, so they both have $d$ components, with the individual elements of $\mathcal{N}_{d}$ as the vertex-sets. For the induction step, clearly $u_{j+1}$ is in the same component of $G_{j}$ as $p_{j+1}$, and $v_{j+1}$ is in the same component of $G_{j}$ as $q_{j+1}$, for each $j=0, \ldots, k-1$, and the result follows immediately.

It follows from Proposition 3.2(2) that

$$
N F_{j}(\sigma)=\left(\begin{array}{c}
k+j \\
j
\end{array}\right) 2^{k-d+1} \mathrm{~L}_{d}^{j} \mathrm{E}_{d} \rho \rho^{\varepsilon},
$$

and then from Proposition 3.2(1) that

$$
N F_{j}(\sigma)=\left(\begin{array}{c}
k+j \\
j
\end{array}\right) 2^{k-d+1} d^{j} \mathrm{E}_{d} \rho \rho^{\varepsilon},
$$


if $j$ is even, and

$$
N F_{j}(\sigma)=\left(\begin{array}{c}
k+j \\
j
\end{array}\right) 2^{k-d+1} d^{j} \mathrm{O}_{d} \rho \rho^{\varepsilon}
$$

if $j$ is odd.

Now, note that $\uplus_{\sigma} \mathcal{N} \mathcal{F}_{j}(\sigma)$, where the disjoint union is over the set of all Hurwitz factorizations $\sigma$, is the set of all near-admissible factorizations with $j$ factors from $\mathcal{F}_{d}$. Moreover, if $\rho \in \mathcal{C} \alpha$ and $b_{\pi} \in \rho$, recall that $\rho \in \mathcal{A}_{\lambda, \mu}$ where $\lambda \cup \mu=\alpha$. The result follows from Proposition 3.2(3), since every $\pi \in \mathfrak{H}_{d}$ has a unique source $b_{\pi}=\rho \in \mathfrak{S}_{d}$. If the Hurwitz factorization $\sigma$ of $\rho$ has genus $g$, and the near-admissible factorizations $a$ in $\mathcal{N F}_{j}(\sigma)$ of $\pi$ have genus $g^{\prime}$, then from conditions HF4 and AF4

$$
2 g+d+l(\lambda)+l(\mu)-2=k, \quad 2 g^{\prime}+2 d+l(\lambda)+2 l(\mu)-2=2 k+j,
$$

and eliminating $k$ between these equations gives $2 g^{\prime}=4 g-2+j+l(\lambda)$.

3.4. The enumeration of admissible factorizations. By eliminating the number of near-admissible factorizations between the two relationships obtained in this section above, we are now able to enumerate admissible factorizations.

Theorem 3.4. (1) For $g \geq 0$, with $j \neq 0$ or $\lambda \neq \emptyset$, and $j \equiv l(\lambda)(\bmod 2)$, then

$$
s_{2 g-1+\frac{1}{2}(j+l(\lambda)), j}(\lambda, \mu)=\left(\begin{array}{c}
k+j \\
j
\end{array}\right) 2^{k-d+1} d^{j} c_{g}(\lambda \cup \mu)
$$

where

$$
2 g+d+l(\lambda)+l(\mu)-2=k .
$$

(2) For $g \geq 0$,

$$
s_{2 g-1,0}(\emptyset, \mu)=2^{l(\mu)-1}\left(4^{g}-1\right) c_{g}(\mu) .
$$

Proof: (1) This follows immediately from Theorems 3.1 and 3.3.

(2) From Theorems 3.1 and 3.3, in the case $j=0$ and $\lambda=\emptyset$, we obtain the equations

$$
t_{2 g-1,0}(\emptyset, \mu)-s_{2 g-1,0}(\emptyset, \mu)=2^{l(\mu)-1} c_{g}(\mu), \quad t_{2 g-1,0}(\emptyset, \mu)=2^{k-d+1} c_{g}(\mu),
$$

with the restriction $2 g+d+l(\mu)-2=k$. Eliminating $t_{2 g-1,0}(\emptyset, \mu)$ between these equations, we obtain

$$
s_{2 g-1,0}(\emptyset, \mu)=\left(2^{k-d+1}-2^{l(\mu)-1}\right) c_{g}(\mu),
$$

and the result follows, since $k-d+1=l(\mu)-1+2 g$. 


\section{GeOMETRIC INTERPRETATIONS OF FACTORIZATIONS}

We now turn to the geometric aspects of the hyperoctahedral analogue of Hurwitz's Problem by brieay recalling some basic facts about ramifed coverings. Let $X$ be a connected Riemann surface of genus $g$ and take $f: \mathrm{X} \rightarrow \mathbb{P}^{1} \mathbb{C}$ as a degree $d$ covering. A point of rami $f$ cation $q$ is called simple for the map $f: \mathrm{X} \rightarrow \mathbb{P}^{1} \mathbb{C}$ if there are two analytic charts $U \subset \mathrm{X}$ and $V \subset f(U) \subset \mathbb{P}^{1} \mathbb{C}, q \in U$, such that in local coordinates $f$ can be represented as the map $z \mapsto z^{2}$, where $z=0$ corresponds to the point $q$.

We say that two pairs $\left(\mathrm{X}_{1} ; f_{1}\right)$ and $\left(\mathrm{X}_{2} ; f_{2}\right)$ are equivalent (here $\sim$ denotes this equivalence relation) if and only if there exists an analytic isomorphism $\varphi: \mathrm{X}_{1} \rightarrow \mathrm{X}_{2}$ such that $f_{2} \varphi=f_{1}$. Let

$$
h_{g}(\alpha)
$$

be the number of equivalence classes of $\sim$ such that the rami $£$ cation at in $£$ nity has poles of orders $\alpha_{1}, \ldots$ (where $\alpha_{1}, \ldots$ are the parts of $\alpha$, with $|\alpha|=d)$. Hurwitz $[\mathrm{H}]$ proved that

$$
h_{g}(\alpha)=c_{g}(\alpha)
$$

by giving a combinatorial encoding of the inequivalent coverings as a transitive factorization in the symmetric group acting on labels for the $d$ sheets.

For admissible factorizations, we consider a generalization of Hurwitz's setting. Let $\tau$ be an involution on $\mathbf{X}$ and consider only those maps $f: \mathbf{X} \rightarrow$ $\mathbb{P}^{1} \mathbb{C}$ of even degree $2 d$ such that $f \tau=f$. Moreover, we say that two triples $\left(\mathrm{X}_{1}, \tau_{1} ; f_{1}\right)$ and $\left(\mathrm{X}_{2}, \tau_{1} ; f_{2}\right)$ are equivalent $\left(\sim_{1}\right.$ denotes this equivalence relation) if and only if there exists an analytic isomorphism $\varphi: X_{1} \rightarrow X_{2}$ such that $f_{2} \varphi=f_{1}$, so the $£$ bres are preserved, and $\tau_{2} \varphi=\varphi \tau_{1}$. This information can be summarized in the commutativity of the following diagram:

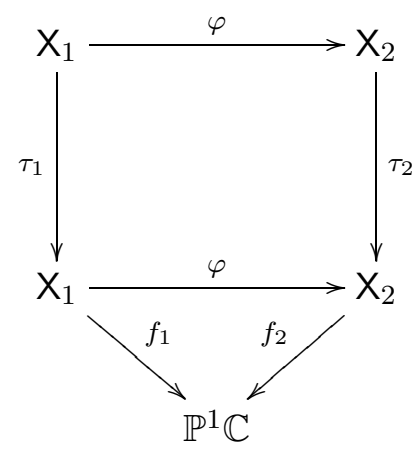

Let $\mathcal{H}\left(\mathfrak{S}_{2}\right)_{2 d, g}$ be the set of equivalence classes $[(\mathrm{X}, \tau ; f)]\left(\right.$ of $\left.\sim_{1}\right)$, where $\mathrm{X}$ is a connected Riemann surface of genus $g, \tau$ is an involution and $f$ is a covering of degree $2 d$ such that: i) $f \tau=f$; ii) $f$ has arbitrary $\tau$-invariant rami $£$ cation over in $£$ nity, and the other branch points are simple points, either $£$ xed by $\tau$, or exchanged in pairs by $\tau$. We call the latter branch 
points doubled. Now consider partitions $\lambda=\left(\lambda_{1}, \ldots\right)$ and $\mu=\left(\mu_{1}, \ldots\right)$, with $|\lambda|=|\mu|=d$. Let

$$
d_{g, j}(\lambda, \mu)
$$

be the number of elements of $\mathcal{H}\left(\mathfrak{S}_{2}\right)_{2 d, g}$ such that the rami $£$ cation over in $£$ nity has poles of orders $\lambda_{1}, \ldots$ that are $£$ xed by $\tau$, poles of orders $\mu_{1}, \ldots$ that are exchanged by $\tau$, with $j$ of the simple branch points $£$ xed by $\tau$, and $k$ of the simple branch points exchanged in pairs by $\tau$.

We now give a particular presentation of $\mathcal{H}\left(\mathfrak{S}_{2}\right)_{2 d, g}$ that leads naturally to admissible factorizations in the hyperoctahedral group. Let $\mathcal{B}$ be the $£-$ nite set of ordered branch points for $f$ (i.e. $\mathcal{B}$ is the branch locus of $f$ ), and let $\pi_{1}\left(\mathbb{P}^{1} \mathbb{C} \backslash \mathcal{B} ; b\right)$ be the fundamental group of $\mathbb{P}^{1} \mathbb{C} \backslash \mathcal{B}$ with respect to a generic point $b$ as base point. We say that two homomorphisms $\beta, \beta^{\prime}: \pi_{1}\left(\mathbb{P}^{1} \mathbb{C} \backslash\right.$ $\mathcal{B} ; b) \rightarrow \widehat{\mathfrak{S}}_{d}$ are equivalent $\left(\sim_{2}\right.$ denotes this equivalence relation) if there exists an element $h \in \mathfrak{H}_{d}$ such that $\beta(\gamma)=h \beta^{\prime}(\gamma) h^{-1}$ for every $\gamma \in \pi_{1}\left(\mathbb{P}^{1} \mathbb{C} \backslash\right.$ $\mathcal{B} ; b)$. With these basic assumptions, it is now possible to translate the information contained in $[(\mathrm{X}, \tau ; f)]$ from geometry to combinatorics. Indeed, let $[(\mathrm{X}, \tau ; f)] \in \mathcal{H}\left(\mathfrak{S}_{2}\right)_{2 d, g}$ and construct $\beta: \pi_{1}\left(\mathbb{P}^{1} \mathbb{C} \backslash \mathcal{B} ; b\right) \rightarrow \widehat{\mathfrak{S}}_{d}$ as described in $[\mathrm{F}]$, where $\mathcal{B}$ is the branch locus of $f$. The image in $\widehat{\mathfrak{S}}_{d}$ of $\pi_{1}\left(\mathbb{P}^{1} \mathbb{C} \backslash \mathcal{B} ; b\right)$ under $\beta$ is called the monodromy group of $f$. In this case, $\left(\mathrm{X}^{\prime}, \tau^{\prime} ; f^{\prime}\right) \sim_{1}(\mathrm{X}, \tau ; f)$ if and only if $\beta \sim_{2} \beta^{\prime}$, because of the commutativity of the above diagram.

Since $f \tau=f$, then the preimage of each point in $\mathbb{P}^{1} \mathbb{C} \backslash \mathcal{B}$ is decomposed into $d$ orbits under the action of $\tau$. In constructing the homomorphism $\beta$, we label the $2 d$ points in $f^{-1}(b)$ with respect to this decomposition, i.e. we mark the points in the $i$-th orbit with labels $i$ and $\hat{i}$ with the ordering $1 \prec \widehat{1} \prec \cdots \prec d \prec \widehat{d}$ along a $£$ bre, thereby labelling the $2 d$ sheets. Then, for each loop around a doubled branch point different from $\infty$, we associate an element $a_{i} \in \mathcal{F}_{d}$. For each loop around the remaining branch points different from in $£$ nity, we associate an element $a_{i} \in \mathcal{G}_{d}$. On the other hand, let us denote the permutation associated with the loop around in£nity by $\pi$. In this way we can uniquely identify a triple in $\mathcal{H}\left(\mathfrak{S}_{2}\right)_{2 d, g}$ with an admissible factorization $a=\left(a_{1}, \ldots, a_{k+j}\right)$ of $\pi$, and hence conclude that

$$
d_{g, j}(\lambda, \mu)=s_{g, j}(\lambda, \mu) .
$$

The geometrical content of the combinatorial conditions AF1-AF4 is as follows. Condition AF1 ensures that the branch points are simple, with $k$ that are doubled, and $j$ that are $£$ xed by $\tau$; condition AF2 ensures that the rami£cation over in£nity has type $(\lambda, \mu)$; condition AF3 ensures that the covering has only one component; condition AF4 ensures, by the RiemannHurwitz formula, that the covering curve has genus $g$.

We can now translate Theorem 3.4, a combinatorial result about factorizations, into the following geometric result, by applying equations (4) and (5). 
Theorem 4.1. (1) For $g \geq 0$, with $j \neq 0$ or $\lambda \neq \emptyset$, and $j \equiv l(\lambda)(\bmod 2)$, then

$$
d_{2 g-1+\frac{1}{2}(j+l(\lambda)), j}(\lambda, \mu)=\left(\begin{array}{c}
k+j \\
j
\end{array}\right) 2^{k-d+1} d^{j} h_{g}(\lambda \cup \mu),
$$

where

$$
2 g+d+l(\lambda)+l(\mu)-2=k .
$$

(2) For $g \geq 0$,

$$
d_{2 g-1,0}(\emptyset, \mu)=2^{l(\mu)-1}\left(4^{g}-1\right) h_{g}(\mu)
$$

\section{A GEOMETRIC PROOF OF THE MAIN RESULT AND RELATED SPECULATIONS}

As shown in [OP], the Hurwitz number $h_{g}(\lambda \cup \mu)$ can be computed in terms of Hodge integrals on moduli spaces of stable maps. As proved in Theorem 4.1, the Hurwitz number $d_{g, j}(\lambda, \mu)$ is related to $h_{g}(\lambda \cup \mu)$. Arguably, this suggests that $d_{g, j}(\lambda, \mu)$ might be computed in the setting of moduli spaces of maps. We formulate a conjecture that originates from a proof of Theorem 4.1 in geometrical terms.

Proof: Let $f: \mathrm{X} \rightarrow \mathbb{P}^{1} \mathbb{C}$ be a degree $2 d$ covering of the Riemann sphere as in Section 4. The action of $\tau$ induces a degree two covering map from $X$ to a Riemann surface, $\mathrm{Y}$. If $\tau$ is Exed-point free, then the map $\phi: \mathrm{X} \rightarrow \mathrm{Y}$ is a topological (unbranched) covering; otherwise, the Exed points of $\tau$ are the rami $£$ cation points of $\phi$. The relationship between the genus of $\mathbf{X}, g(\mathbf{X})$, and that of $\mathrm{Y}, g(\mathrm{Y})$, is given by the Riemann-Hurwitz formula, and is

$$
2-2 g(\mathrm{X})=2(2-2 g(\mathrm{Y}))-j-l(\lambda) .
$$

In addition to $\phi$, the covering $f$ induces a degree $d$ covering $f^{\prime}: Y \rightarrow \mathbb{P}^{1} \mathbb{C}$. The rami $£$ cation points of $f^{\prime}$ are the images (under $\phi$ ) of the $l(\lambda)$ points Exed by $\tau$, as well as the images (under $\phi$ ) of the $2 k$ rami£cation points lying over the branch doubled points of $f$. Applying the Riemann-Hurwitz formula to $f^{\prime}$, we have:

$$
2-2 g(\mathrm{Y})=d-k+l(\lambda)+l(\mu) .
$$

Note that combining (6) and (7) results in applying Riemann-Hurwitz formula to the covering $f$. Moreover, the following diagram is commutative:

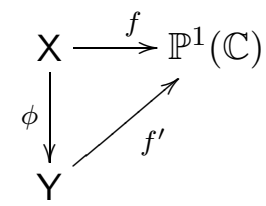

Furthermore, $\sim_{1}$-equivalent coverings to $f$ yield coverings of the Riemann sphere which are equivalent to $f^{\prime}$ with respect to the usual equivalence of coverings. Therefore, it is possible to enumerate equivalence classes of $\mathfrak{S}_{2}$-invariant coverings through the Hurwitz numbers $h_{g(\mathrm{Y})}$. There are two cases. 
First, let us consider the case when $\tau$ is Exed-point free, i.e., $j=0$ and $\lambda=\emptyset$. By standard facts in algebraic topology (see, e.g., [Hat], p. $71 \mathrm{ff}$.), the fundamental group $\pi_{1}(\mathrm{X})$ is an index two subgroup of the fundamental group $\pi_{1}(\mathrm{Y})$. Thus, enumerating all degree two (unbranched) coverings from $X$ to $Y$ is tantamount to enumerating all index two subgroups of $\pi_{1}(\mathrm{Y})$. These correspond to homomorphisms from $\pi_{1}(\mathrm{Y})$ to the cyclic group of order two. As a result, there are $2^{2 g(\mathrm{Y})}$ coverings of a Riemann surface of genus $g(\mathrm{Y})$, all but one of which are connected. Note that in this case (6) gives $g(\mathrm{X})=2 g(\mathrm{Y})-1$. Therefore, there are $\left(4^{g(\mathrm{Y})}-1\right) h_{g(\mathrm{Y})}(\mu)$ degree $2 d$ maps from a Riemann surface $\mathbf{X}$ of genus $g(\mathbf{X})=2 g-1$ with $\mathfrak{S}_{2}$-invariant rami $£$ cation over $\infty$ given by the partition $2 \mu$. Any such map is actually taken into account by our enumeration process if we also assign 'hats' to all labels that appear in the cycles of $\mu$. This can be done in $\frac{1}{2} 2^{l(\mu)}$ ways. Indeed, hatted and unhatted symbols are interchanged via conjugation by $\varepsilon$, which yields pairs of equivalent coverings of degree $2 d$. Thus Theorem 4.1(2) follows.

Second, let us assume $\tau$ is not a Exed-point free involution. Let $f^{\prime}$ : $\mathrm{Y} \rightarrow \mathbb{P}^{1} \mathbb{C}$ be a degree $d$ covering with $k$ simple rami $£$ cation points and $2 d-l(\lambda)-l(\mu)$ points over $\infty$. For a branched degree two covering of $\mathrm{Y}$ we need to choose $j$ extra points such that $j \equiv l(\lambda) \bmod 2$ (see (6)). Clearly, they can be chosen in $d^{j}$ ways. Moreover, such points are chosen so that $j$ among the $j+k$ points are not doubled branch points of $f$. Analogously to the previous case, there are $2^{2 g(\mathrm{Y})}$ branched covering of degree two with $\frac{1}{2}(j+$ $l(\lambda))$ rami $£$ cation points, all of which are connected since $j \neq 0$. In order to enumerate the degree $2 d$ coverings induced by $f^{\prime}$, we still need to assign 'hats' to the labels that appear in the cycles of $\lambda \cup \mu$. As above, this can be done in $2^{l(\lambda)+l(\mu)-1}$ ways. Therefore, we have:

$$
d_{g(\mathrm{X}), j}(\lambda, \mu)=\left(\begin{array}{c}
k+j \\
j
\end{array}\right) d^{j} 2^{2 g(\mathrm{Y})+l(\lambda)+l(\mu)-1} h_{g(\mathrm{Y})}(\lambda \cup \mu) .
$$

If we set $g(\mathrm{Y})=g$, then (6) yields $g(\mathrm{X})=2 g-1+\frac{1}{2}(j+l(\lambda))$. Thus, Theorem 4.1(1) follows; indeed, by (7)

$$
2 g+l(\lambda)+l(\mu)-1=k-d+1 .
$$

To state our conjecture, we need to introduce some conventional notation.

Let $\mathcal{M}_{g}$ be the moduli space of smooth genus $g$ curves, $g \geq 2$, over $\mathbb{C}$. We recall that $\mathcal{M}_{g}$ has an orbifold structure which is given by the action of the mapping class group $\Gamma_{g}$ on the Teichmüller space $\mathcal{T}_{g}$. The reader is referred to, e.g., [Har] for the de£nitions of $\Gamma_{g}$ and $\mathcal{T}_{g}$.

Fix an order two subgroup $G \cong \mathfrak{S}_{2}$ with an embedding in $\Gamma_{g}$. Assume $G$ is generated by an involution $\tau$ with at least $l(\lambda)$ Exed points. Note that $\tau$ 
may have $j$ additional $£$ xed points, $j \geq 0$. Let $g^{\prime}$ be a non negative integer such that $2-2 g=2\left(2-2 g^{\prime}\right)-j-l(\lambda)$. Set, further, $w^{\prime}=2 g^{\prime}+2 d+$ $l(\lambda)+l(\mu)-2$. As in [OP], $\S 7.3$, let $\overline{\mathcal{M}}_{g^{\prime}}(\lambda \cup \mu)$ be the closure in $\overline{\mathcal{M}}_{g^{\prime}}\left(\mathbb{P}^{1} \mathbb{C}, d\right)$ of $\mathcal{M}_{g^{\prime}}(\lambda \cup \mu)$, which parametrizes degree $d$ stable maps with rami£cation given by $\lambda \cup \mu$ over $\infty$.

Conjecture 5.1. 1 . There exists a (coarse) moduli space $\overline{\mathcal{M}}_{g}^{G}(\lambda, \mu)$ that contains a (Zariski) open set $\mathcal{M}_{g}^{G}(\lambda, \mu)$ satisfying

i) $\mathcal{M}_{g}^{G}(\lambda, \mu)$ parametrizes up to isomorphism pairs $(C, f)$, where $C$ is a smooth genus $g$ curve and $f: C \rightarrow \mathbb{P}^{1} \mathbb{C}$ is a degree $2 d$ covering map;

ii) $f \circ \tau=f$;

iii) $f$ has ramifcation profle prescribed by $\lambda$ and $\mu$ over $\infty .^{1}$

2. There is a well-de£ned morphism $p: \overline{\mathcal{M}}_{g}^{G}(\lambda, \mu) \rightarrow \overline{\mathcal{M}}_{g^{\prime}}(\lambda \cup \mu)$. Denote by $B r$ the composition br $\circ p$, where $b r: \overline{\mathcal{M}}_{g^{\prime}}(\lambda \cup \mu) \rightarrow \mathbb{P}^{w^{\prime}} \mathbb{C}$ is the branch morphism deEned in [OP].

3. The moduli space $\overline{\mathcal{M}}_{g}^{G}(\lambda, \mu)$ has a virtual fundamental class $\left[\overline{\mathcal{M}}_{g}^{G}(\lambda, \mu)\right]^{\text {vir }}$.

4. Let $\xi_{p}$ denote (the Poincare dual of) the point class of

$$
\begin{gathered}
L_{m}=\left\{D+m[p]: D \in \operatorname{Sym}^{w^{\prime}-m}\left(\mathbb{P}^{1} \mathbb{C}\right) \subset \operatorname{Sym}^{w^{\prime}}\left(\mathbb{P}^{1} \mathbb{C}\right) \cong \mathbb{P}^{w^{\prime}}\right\}, \\
m:=d-l(\lambda)-\lambda(\mu),
\end{gathered}
$$

where $S y m^{w^{\prime}-m}\left(\mathbb{P}^{1} \mathbb{C}\right)$ is the symmetric product of $\mathbb{P}^{1} \mathbb{C}$.

Then the following hold:

a) for $g \geq 0$, with $j \neq 0$ or $\lambda \neq \emptyset$, and $j \equiv l(\lambda)(\bmod 2)$, we have

$$
\int_{\left[\overline{\mathcal{M}}_{g}^{G}(\lambda, \mu)\right]^{v i r}} B r^{*}\left(\xi_{p}\right)=\left(\begin{array}{c}
g^{\prime}+l(\lambda)+l(\mu)+j-1 \\
j
\end{array}\right) 2^{g^{\prime}+l(\lambda)+l(\mu)-d+j} d^{j} h_{g^{\prime}}(\lambda \cup \mu) ;
$$

b) for $g \geq 0$, we have

$$
\int_{\left[\overline{\mathcal{M}}_{g}^{G}(\lambda, \mu)\right]^{v i r}} B r^{*}\left(\xi_{p}\right)=2^{l(\mu)-1)}\left(4^{g^{\prime}}-1\right) h_{g^{\prime}}(\mu) .
$$

Conjecture 5.1 stems from the following remarks on the geometrical proof of Theorem 4.1. Any covering $f^{\prime}: \mathrm{Y} \rightarrow \mathbb{P}^{1} \mathbb{C}$ in Diagram 8 is enumerated by the classical Hurwitz number, where $g(\mathrm{Y})=g^{\prime}$. Fixing $\tau$ and its topological action (i.e., an embedding of $G$ in $\Gamma_{g}$ ) allows one to construct a degree two covering $\phi: \mathrm{X} \rightarrow \mathrm{Y}$, as well as a degree $2 d$ covering map $f: \mathrm{X} \rightarrow \mathbb{P}^{1} \mathbb{C}$. This suggests the existence of a moduli space, $\overline{\mathcal{M}}_{g}^{G}(\lambda, \mu)$, as in Conjecture 5.1, 1 .

\footnotetext{
${ }^{1}$ Condition ii) implies that the rami $£$ cation points of $f$ are either $£$ xed or exchanged by $\tau$. Since $\tau$ has $j+l(\lambda)$ £xed points, the partition $\mu$ describes the pairs of $£$ xed points exchanged by $\tau$.
} 
The generic point of such a moduli space is a covering with simple rami $£$ cation points. Some of them are £xed by $\tau$; some others are exchanged by the involution.

The way of passing from $f^{\prime}: Y \rightarrow \mathbb{P}^{1} \mathbb{C}$ to $f: X \rightarrow \mathbb{P}^{1} \mathbb{C}$ via $\phi$ leads us to the second part of Conjecture 5.1. The image under $p$ of a generic point $(\mathrm{X}, f) \in \overline{\mathcal{M}}_{g}^{G}(\lambda, \mu)$ should be the element $\left(\mathrm{Y}, f^{\prime}\right)$. Moreover, the morphism $B r$ leads us to conjecture an intersection theoretic computation of $d_{g, j}(\lambda, \mu)$. This requires that a virtual fundamental class of $\overline{\mathcal{M}}_{g}^{G}(\lambda, \mu)$ exists.

As shown in [OP], the classical Hurwitz number can be viewed as the degree of the branch morphism $b r$. In fact, Bertini's Theorem applied to a generic divisor $D+m[p]$ in $L_{m}$ implies that the intersection points in $b r^{-1}(D+m[p]) \cap \mathcal{M}_{g^{\prime}}(\lambda \cup \mu)$ are exaclty the coverings enumerated via $h_{g^{\prime}}(\lambda \cup$ $\mu)$. In other words,

$$
h_{g^{\prime}}(\lambda \cup \mu)=\int_{\left[\overline{\mathcal{M}}_{g^{\prime}}(\lambda \cup \mu)\right]^{v i r}} b r^{*}\left(\xi_{p}\right) .
$$
b).

Similar arguments applied to $B r$ give evidence to Conjecture 5.1, 4. a),

Note that Conjecture 5.1, 4. a), b) compute the degree of the morphism $B r$. Furthermore, we recall that $h_{g^{\prime}}(\lambda \cup \mu)$ is the degree of $b r$. Thus, our conjecture could give some information on the degree of the morphism $p$.

\section{ACKNOWLEDGEMENTS}

We would like to thank John Harer for suggesting the problem that motivated this research. This research was supported by Discovery Grants from the Natural Sciences and Engineering Research Council of Canada individually to IPG and DMJ. GB would like to thank the University of Waterloo for Enancial support and warm hospitality during his stay in Fall 1998.

\section{REFERENCES}

[ELSV] T. EKEDAHL, S. LANDO, M. SHAPIRO AND A. VAINSHTEIN, Hurwitz numbers and intersections on moduli spaces of curves, Invent. Math. 146 (2001), 297-327.

[F] W. FUlTON, Hurwitz schemes and irreducibility of moduli of algebraic curves, Ann. Math. 90 (1969), 542-575.

[GJ1] I.P. GOULDEN AND D.M. JACKSON, Transitive factorisations into transpositions and holomorphic mappings on the sphere, Proc. A.M.S. 125 (1997), 51-60.

[GJ2] I.P. GOULDEN AND D.M. JACKSON, A proof of a conjecture for the number of ramifed coverings of the sphere by the torus, J. Combinatorial Theory (A) 88 (1999), 246-258.

[Har] J. HARER, The cohomology of the moduli space of curves, in: Theory of moduli (Montecatini Terme, 1985), 138-221, Lecture Notes in Math., 1337, Springer, Berlin, 1988.

[Hat] A. HAtcher, Algebraic topology, Cambridge University Press, Cambridge, 2002. 
[H] A. HuRwitz, Ueber Riemann'sche Flächen mit gegebenen Verzweigungspunkten, Matematische Annalen 39 (1891), 1-60.

[OP] A. OKOUNKOV, R. PANDHARIPANDE, Gromov-Witten theory, Hurwitz numbers, and matrix models, I, AG-0101147.

[V2] R. VAKIL, Enumerative geometry of plane curves of low genus, AG-9803007. 